\title{
Cerebral ventriculitis associated with Achromobacter xylosoxidans
}

\author{
SHIRO SHIGETA, YOSHIO YASUNAGA, KEN HONZUMI, HIROKO OKAMURA, \\ ROKURO KUMATA, AND SHINICHIRO ENDO
}

From the Central Clinical Laboratory and 2nd Department of Surgery, Fukushima Medical College, Fukushima, Japan

SUMMARY Six patients in the neurosurgical ward of Fukushima Medical College Hospital suffering from ventriculitis due to Achromobacter xylosoxidans infection had undergone craniotomy or cranial trepanation before the infection. The strains of $A$. xylosoxidans isolated from the patients $O$ were resistant to streptomycin, ampicillin, cephaloridine, gentamicin, and colistin. They were also resistant to chlorhexidine digluconate (Hibitane) in a concentration of $2 \%$. When a study of the chlorhexidine used in the hospital was carried out four strains of $A$. xylosoxidans were isolated from 20 containers of chlorhexidine solution in the surgical ward but not from those in the operating theatre.

Achromobacter xylosoxidans, a nonfermentative, Gram-negative, peritrichous rod, was named and described by Yabuuchi and Ohyama (1971) in isolates from patients with chronic otitis media. The homogeneity of $A$. xylosoxidans was established as a result of Yabuuchi's subsequent study of 55 strains in which the uniformities of flagellar morphology, biochemical reaction, and percentage of guanine and cytosine mole in deoxyribonucleic acid were confirmed (Yabuuchi et al., 1974). Although A. xylosoxidans has been recovered from a variety of human clinical sources, including blood, bile, and cerebrospinal fluid (CSF), its role as the primary aetiological agent of meningitis was proved and reported by us as a first case report (Shigeta et al., 1974). This paper describes the recovery of $A$. xylosoxidans from the CSF of six patients with ventricular infection after neurosurgical operations.

\section{Material and methods}

BACTERIOLOGICAL SURVEY

All patients admitted to the neurosurgical ward of Fukushima Medical College Hospital, from whom A. xylosoxidans and other species of organisms were isolated from the CSF during the period May 1973 to February 1975, were included in this survey. CSF was the sole material for culture, and isolates

Received for publication 17 November 1976 were identified using triple-sugar iron (TSI) agar, oxidation, and fermentation of sugars and decarboxylase media devised by Møller (1955).

In addition, production of water- or chloroformsoluble pigments, nitrate reduction, motility, and flagellar morphology were determined. The programme of identification was essentially that of Yabuuchi et al. (1974). Organisms were considered to cause infection only when a pure culture of A. xylosoxidans was obtained from samples of CSF 8 taken by ventricular puncture or drained from the ventricles, and when there was clinical evidence of meningitis. Newly isolated strains of $A$. xylosoxidans, Pseudomonas aeruginosa, Escherichia coli, and $₹$ Klebsiella pneumoniae were subcultured on Dorset's 은 egg medium (Nissui Ltd, Tokyo) and stored for one $>$ to three months. Before testing for sensitivity and resistance of the organisms, all strains were in- $\bar{N}$ oculated in brain-heart infusion (BHI) broth and $\%$ cultured at $37^{\circ} \mathrm{C}$ for 24 hours.

SENSITIVITY TO ANTIBIOTICS

Sensitivity to antibiotics was tested using the single

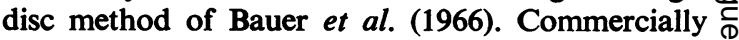
purchased discs (Showa Pharmaceuticals Ltd, $\stackrel{?}{+}$ Tokyo) and Müller-Hinton agar (BBL) were used in the test.

RESISTANCE TO CHEMICAL DISINFECTION The resistance of $A$. xylosoxidans to disinfection was 
tested by the addition of about $10^{8}$ organisms to $1 \mathrm{ml}$ of several dilutions of disinfectant. As a control, the same number of organisms was added to distilled water without disinfectant. The mixtures of the organisms and disinfectant or distilled water were kept at room temperature for 10 minutes and were then diluted to $10^{-2}$ with sterile saline, and $0.01 \mathrm{ml}$ of the dilution was inoculated in BHI broth.

In each test $4 \times 10^{3}$ to $3 \times 10^{4}$ organisms were inoculated in broth, and all test cultures were divided into two sets and then incubated at $25^{\circ} \mathrm{C}$ and $37^{\circ} \mathrm{C}$. After 48 hours' incubation the cultures were examined for growth of organisms, and the resistance of the organisms was judged by their survival in the dilution of disinfectant after treatment. In another experiment the numbers of survivors were assessed by inoculation on a BHI agar plate after treatment.

\section{INVESTIGATION FOR SOURCE OF INFECTION}

Investigation of the chlorhexidine solution prepared in the operating theatre and in the surgical ward was carried out by inoculating a 1-ml aliquot of sample from the containers into $10 \mathrm{ml}$ of BHI broth. Duplicate broth tubes were used and divided into two sets and were then incubated at $25^{\circ} \mathrm{C}$ and $37^{\circ} \mathrm{C}$ for 10 days. On the last day of culture, one drop of broth was subcultured on BHI agar containing $5 \%$ sheep erythrocytes and was then incubated at $37^{\circ} \mathrm{C}$ for $\mathbf{4 8}$ hours to isolate the contaminating organisms.

\section{Results}

\section{CASE REPORTS}

\section{Case 1}

A girl aged 9 was admitted to the neurosurgical ward on 7 March, 1973 for operation on an arachnoid cyst in the occipital region. On the tenth postoperative day she underwent a ventriculoperitoneal shunt to reduce the pressure of the CSF in the ventricles. On 19 May she developed fever $\left(39^{\circ} \mathrm{C}\right)$ with continuous headache, frequent vomiting, and stiffness of the neck. On 29 May, ventricular puncture was performed at the site of the ventricular tap which connected the ventricles to the peritoneal shunt. The puncture yielded purulent CSF, and $A$. xylosoxidans was isolated from the fluid in pure culture. The patient received injections of chloramphenicol ( $1 \mathrm{~g}$ daily) and the infection improved temporarily but later recurred three times in September, November, and December of the same year. Three of the four relapses were associated with $A$. xylosoxidans infection. Sera tested for the agglutinating antibody to the isolates on 15 June and 6 September had titres of 1 in 320 and 1 in 640.

\section{Case 2}

This 6-month-old boy had a haematoma in the occipital region after birth in October 1973. A swelling gradually developed associated with redness and fever. In February 1974 a cerebral abscess was diagnosed. Incision of the abscess yielded a purulent fluid from which Proteus mirabilis was recovered. After the incision, two drains were inserted in the abscess and in the corresponding ventricles of the cerebrum. In spite of extensive chemotherapy with daily injections of cephaloridine $(1.0 \mathrm{~g})$, the infection persisted for two months and on 16 April, A. xylosoxidans was isolated in pure culture not only from pus in the abscess but also from CSF drained from the ventricle. The organism was sensitive to chloramphenicol, ampicillin, carbenicillin, and nalidixic acid. Daily injections of chloramphenicol $(500 \mathrm{mg})$ were continued for two weeks and he recovered from the infection.

\section{Case 3}

A 9-year-old boy with hydrocephalus had a cranial trepanation, and a drain was inserted in the ventricle on 10 July 1973. After the operation he was pyrexic $\left(38.4^{\circ} \mathrm{C}\right)$ and three days after the operation $A$. xylosoxidans was isolated from CSF drained from the ventricle.

\section{Case 4}

This 8-year-old boy with a thalamic tumour had a ventriculoperitoneal shunt operation. After the operation he became pyrexic. One month later, a ventricular puncture yielded a turbid CSF from which $A$. xylosoxidans was isolated. After a 6-day course of chloramphenicol ( $500 \mathrm{mg}$ daily), there was no growth of $A$. xylosoxidans but Serratia marcescens was isolated on culture.

\section{Case 5}

A 63-year-old man had a radical operation for multiple cerebral aneurysms performed on 10 July 1974. After the operation a drain was inserted in the ventricle. On the sixth day after operation, he had a fever of $39^{\circ} \mathrm{C}$ and $A$. xylosoxidans was isolated from the CSF in pure culture; on the following day the same organism was again isolated. These organisms were shown to be sensitive to tetracycline, chloramphenicol, and nalidixic acid but completely resistant to gentamicin, colistin, kanamycin, carbenicillin, and cephaloridine. He made a complete recovery from the infection after a two-week course of chloramphenicol injections ( $1.0 \mathrm{~g}$ daily). 
Case 6

A man aged 49 was admitted to the surgical ward with a cerebral tumour on 9 September 1974. On 5 February 1975 he underwent craniotomy, and part of the tumour, shown histologically to be a meningioma, was resected. After the operation he had a fever of $39^{\circ} \mathrm{C}$ which continued for several days. On 11 February ventricular puncture yielded a turbid CSF from which $A$. xylosoxidans was isolated.

\section{INCIDENCE OF BACTERIAL VENTRICULITIS}

\section{IN THE NEUROSURGICAL WARD}

From May 1973 to February 1975,11 patients were admitted to the neurosurgical ward of Fukushima Medical College Hospital suffering from bacterial ventriculitis. Craniotomy or cranial trepanation had been performed on all the patients for resection of a tumour or incision of an abscess cavity. Each patient also had a ventriculoperitoneal shunt or drain from the ventricle.

Six of the 11 patients were infected with $A$. xylosoxidans, and the details of those cases have been cited above. Other species of organisms recovered from the CSF of these patients were Enterobacter, Staphylococcus aureus, Staphylococcus epidermidis, $P$ s. aeruginosa, $P$. mirabilis, $S$. marcescens, Acinetobacter calcoaceticus, and $K$. pneumoniae.

Some patients relapsed with the same infection or with an infection caused by different species of organisms. If such relapses are considered as individual cases, there were 18 cases of ventriculitis among 11 patients during the period of the survey. The causative organisms in these cases are shown in Table 1. Eight of 18 were A. xylosoxidans and three others were nonfermentative rods (2 $A$. calcoaceticus and 1 Ps. aeruginosa). Eleven $A$. xylosoxidans strains were isolated from the CSF of these patients and some characteristics of the isolated strains and type strain ATCC-27061 of

Table 1 Causative organisms in 18 cases of bacterial meningitis

\begin{tabular}{llc}
\hline Causative organism & \multicolumn{2}{l}{ Cases of infection* } \\
\cline { 2 - 3 } & No. & $\%$ \\
\hline Achromobacter xylosoxidans & 8 & $44 \cdot 4$ \\
Enterobacter spp & 2 & $11 \cdot 1$ \\
Acinetobacter calcoaceticus & 2 & $11 \cdot 1$ \\
Serratia marcescens & 1 & $5 \cdot 6$ \\
Staphylococcus aureus & 1 & $5 \cdot 6$ \\
Staphylococcus epidermidis & 1 & $5 \cdot 6$ \\
Pseudomonas aeruginosa & 1 & $5 \cdot 6$ \\
Proteus mirabilis & 1 & $5 \cdot 6$ \\
Klebsiella pneumoniae & 1 & $5 \cdot 6$ \\
Total & 18 & \\
\hline
\end{tabular}

* Relapses of infection with the same or different species of organisms were considered as individual cases.

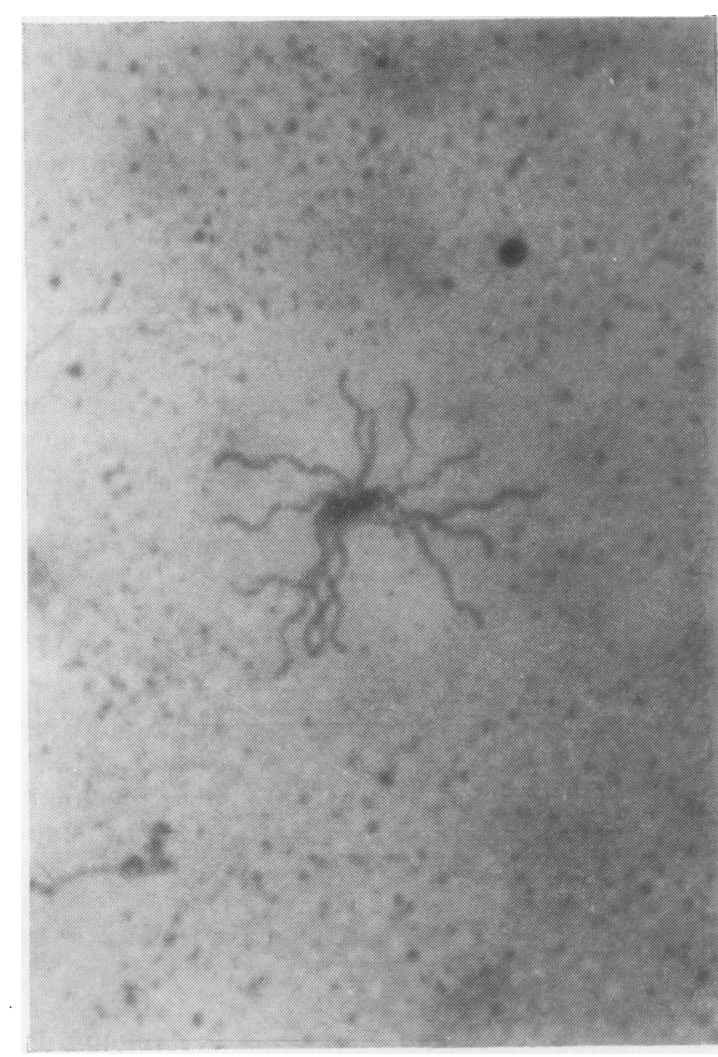

Figure Achromobacter xylosoxidans F-1482 isolated from cerebrospinal fluid of case 1. Leifson's flagellar staining after formalin fixation. $\times 1000$.

A. xylosoxidans are shown in Table 2 and the Figure.

SENSITIVITY OF A. XYLOSOXIDANS TO

ANTIBIOTICS

Eleven strains of $A$. xylosoxidans isolated from CSF 은 were tested for sensitivity to antibiotics. As shown $D$ in Table 3, all strains were resistant to streptomycin, cephaloridine, and ampicillin. Ten strains were $N$ resistant to gentamicin and eight strains were resist- $\circ$ ant to colistin. However, more than half of all the $N$ strains were sensitive to tetracycline, chlorampheni- $\underset{\omega}{\omega}$ col, nalidixic acid, and carbenicillin.

\section{RESISTANCE OF A. XYLOSOXIDANS TO} CHEMICAL DISINFECTION

It was conceivable that the $A$. xylosoxidans infection $D$ in these patients might have been due to the resistance of organisms to, and failure of, chemical $\stackrel{\mathbb{D}}{\circ}$ disinfection. In this hospital, chlorhexidine (Hibitane) $\stackrel{\mathbb{D}}{\triangle}$ was the most popular disinfectant for washing the 
Table 2 Characteristics of the type strain of A. xylosoxidans and of 11 isolated strains

\begin{tabular}{lcc}
\hline Substrates and tests & Type strain & No. of posi- \\
& ATCC-27061 & tive strains \\
\hline Gram negative, rod shaped & + & 11 \\
Motility & + & 11 \\
Peritrichous flagella & + & 11 \\
Growth on NAC agar & + & 11 \\
Indophenol oxidase & + & 11 \\
Citrate, Simmons & + & 11 \\
OF-glucose medium open, acid & + & $11_{3-4}$ \\
OF-glucose medium sealed, acid & - & 0 \\
Oxidative acid production from & & \\
Fructose & - & $2,-7$ \\
Mannose & - & 2, \\
Galactose & - & 0 \\
D-arabinose & - & 0 \\
Xylose & + & $11_{1-3}$ \\
Maltose & - & 0 \\
Ethanol (3\%) & + & $11_{3}-4$ \\
L-lysine decarboxylase & - & 0 \\
L-arginine dihydrolase & - & 9 \\
Amidase for propionate & + & \\
\hline
\end{tabular}

Subscripts indicate days required for positive reaction.

*Used OF basal medium (Difco) containing $1 \%$ carbohydrate.

Table 3 Results of antibiotic sensitivity test on 11 strains of A. xylosoxidans

\begin{tabular}{lrlr}
\hline Antibiotics & \multicolumn{3}{c}{$\begin{array}{l}\text { Sensitive } \\
\text { strains }\end{array}$} \\
\cline { 2 - 4 } & \multicolumn{4}{l}{ No. } \\
\hline Tetracycline & $200 \mu \mathrm{g}$ & 7 & $63 \cdot 6$ \\
Chloramphenicol & $100 \mu \mathrm{g}$ & 8 & $72 \cdot 7$ \\
Streptomycin & $50 \mu \mathrm{g}$ & 0 & $0 \cdot 0$ \\
Kanamycin & $50 \mu \mathrm{g}$ & 5 & $45 \cdot 5$ \\
Cephaloridine & $30 \mu \mathrm{g}$ & 0 & $0 \cdot 0$ \\
Ampicillin & $30 \mu \mathrm{g}$ & 0 & $0 \cdot 0$ \\
Carbenicillin & $30 \mu \mathrm{g}$ & 8 & $72 \cdot 7$ \\
Gentamicin & $30 \mu \mathrm{g}$ & 1 & $9 \cdot 1$ \\
Colistin & $150 \mathrm{units}$ & 3 & $27 \cdot 3$ \\
Nalidixic acid & $50 \mu \mathrm{g}$ & 9 & $81 \cdot 8$ \\
\hline
\end{tabular}

Showa sensitivity disk was used.

surgeon's hands and for the preparation of the patient's skin before operation. Therefore the resistance of $A$.xylosoxidans to chlorhexidine and two other disinfectants, ie, benzalkonium chloride (Osban, Takeda Chemical Industries Ltd, Osaka) and Cresol solution (Dainippon Pharmaceutical Ltd, Tokyo) was examined. Seven of $11 \mathrm{~A}$. xylosoxidans strains were resistant to $2.0 \%$ chlorhexidine and three other strains were resistant to $1.0 \%$ chlorhexidine after 10 minutes' treatment (Table 4). This
Table 5 Percentage of surviving organisms in $0.1 \%$ chlorhexidine

\begin{tabular}{lccccc}
\hline Organism & Strain No. & \multicolumn{5}{c}{ Time for treatment (minutes) } \\
\cline { 2 - 6 } & & 1 & 5 & 10 & 20 \\
\hline A. xylosoxidans & 1948 & 32.3 & 32.0 & 27.5 & 18.7 \\
A. xylosoxidans & 4473 & 35.0 & 24.7 & 25.0 & 27.5 \\
Staph. aureus* & & 1.3 & 0.7 & 0.07 & $<0.05$ \\
E. coli* & & $<0.05$ & $<0.05$ & $<0.05$ & $<0.05$ \\
Ps. aeruginosa* $^{*}$ & & $<0.05$ & $<0.05$ & $<0.05$ & $<0.05$ \\
\hline
\end{tabular}

*Isolates from clinical specimens.

dose of chlorhexidine is 10 times that of the concentration $(0.1 \%)$ used in this hospital. On the other hand, $P$ s. aeruginosa, $E$. coli, and $K$. pneumoniae were completely destroyed by benzalkonium chloride and Cresol at the concentrations used in this hospital $(1.0 \%$ and $3.0 \%$, respectively). In order to examine the survival of $A$. xylosoxidans in the dilution of chlorhexidine used in the hospital representative strains of $A$. xylosoxidans and other organisms were treated with $0.1 \%$ chlorhexidine and titrated, following the time course shown in Table 5. The results showed that almost one-quarter of the A. xylosoxidans organisms survived in the chlorhexidine after 10 minutes' treatment.

\section{ISOLATION OF A. XYLOSOXIDANS FROM \\ CHLORHEXIDINE SOLUTION}

In order to investigate the source of the infection, chlorhexidine used in the vicinity of patients was tested for contaminants. In April 1975 (2 months after the last outbreak of infection) and in February 1976 , a study was carried out in the operating theatre and in the surgical ward for the isolation of organisms from each washbasin. One-tenth percent chlorhexidine had been prepared with sterilised distilled water in the operating theatre and with unsterilised tap water in the surgical ward. In both areas samples were taken from 10 to 20 separate basins. The results are shown in Table 6. Four $A$. xylosoxidans strains were isolated from basins in the surgical ward. One $P$. cepacia strain was isolated from a bowl used for the disinfection of surgical instruments. No organisms were isolated from basins in the operating theatre.

Table 4 Resistance of A. xylosoxidans and other organisms to concentrations of chlorhexidine

\begin{tabular}{|c|c|c|c|c|c|c|}
\hline \multirow[t]{2}{*}{ Organism } & \multirow{2}{*}{$\begin{array}{l}\text { No. of strains } \\
\text { tested }\end{array}$} & \multicolumn{5}{|c|}{ No. of strains resistant to chlorhexidine with a concentration of: } \\
\hline & & $2.0 \%$ & $1 \cdot 0 \%$ & $0.1 \%$ & $0.01 \%$ & $<0.01 \%$ \\
\hline $\begin{array}{l}\text { A. xylosoxidans } \\
\text { Ps. aeruginosa } \\
\text { E. coli } \\
\text { K. pneumoniae }\end{array}$ & $\begin{array}{l}11 \\
5 \\
2 \\
2\end{array}$ & $\begin{array}{l}7 \\
0 \\
0 \\
0\end{array}$ & $\begin{array}{l}3 \\
0 \\
0 \\
0\end{array}$ & $\begin{array}{l}1 \\
0 \\
0 \\
0\end{array}$ & $\begin{array}{l}\mathbf{0} \\
\mathbf{2} \\
\mathbf{0} \\
\mathbf{0}\end{array}$ & $\begin{array}{l}\mathbf{0} \\
\mathbf{3} \\
\mathbf{2} \\
\mathbf{2}\end{array}$ \\
\hline
\end{tabular}


Table 6 Isolation of A. xylosoxidans from $0.1 \%$ chlorhexidine in washbasins and other containers

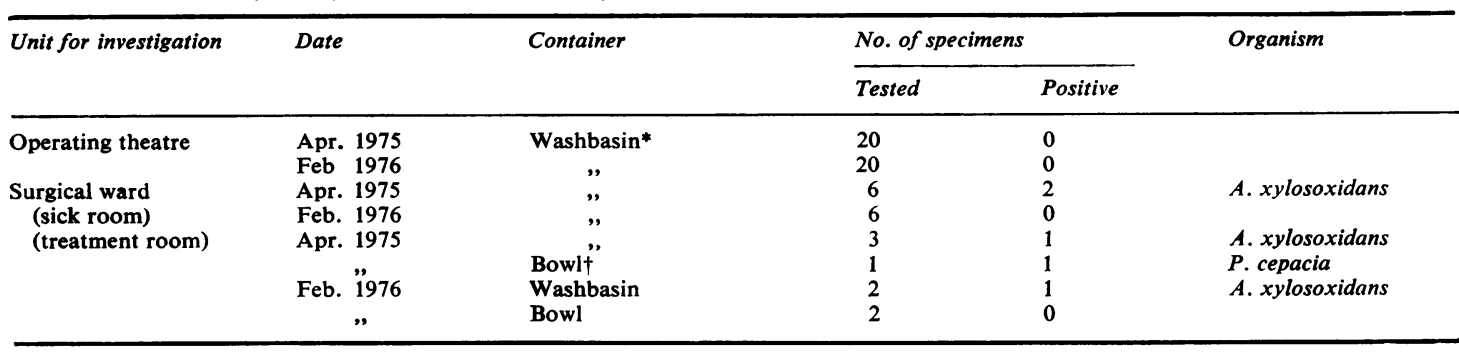

*In the operating theatre chlorhexidine was changed in the basin after each hand washing but in the surgical ward it was changed only twice a day.

†The bowl contained chlorhexidine for the disinfection of used surgical instruments.

\section{Discussion}

The case reports presented concern bacterial ventriculitis due to $A$. xylosoxidans infection. All patients suffered from the infection after craniotomy or cranial trepanation had been performed. $A$. xylosoxidans is the pathogen in this context because it was the only species isolated from the CSF in these patients during the relevant ventriculitis. In each case $A$. xylosoxidans was the sole aerobic isolate of cultured organisms and the clinical process of infection corresponded well with the isolation of this agent. In case 1 , the patient relapsed three times with ventriculitis associated with the recovery of A. xylosoxidans. In addition, the patient's serum agglutinated the isolate up to a dilution of 1 in 640 . Except in case 1, the duration of infection with $A$. xylosoxidans was relatively short and patients recovered quickly with appropriate chemotherapy.

Ten of 11 strains of $A$. xylosoxidans isolated were resistant to $1.0 \%$ chlorhexidine, which was 10 times the concentration of that used to disinfect the surgeon's and nurse's hands in the hospital. Although the study of chlorhexidine used in this hospital was carried out two months after the last infection with $A$. xylosoxidans the organisms still persisted in the surgical ward and were isolated from the washbasins. The observations cited above suggest that the infection described was of nosocomial origin.

Chlorhexidine, one of the derivatives of bisdiguanide, was reported to be effective on several kinds of microorganisms, including Gram-positive and -negative bacteria (Davies et al., 1954; Calman and Murray, 1956). Pseudomonas cepacia, recently documented as a cause of nosocomial infection (Ederer and Matsen, 1972), was reported to be resistant to Savlon, which contained chlorhexidine and cetrimide (Bassett et al., 1970). Alcaligenes fecalis which is similar in morphology and biochemical characteristics to $A$. xylosoxidans, was also reported to be resistant to chlorhexidine (Kim et al., 1973). Recently, analytical work on the fatty $\vec{v}$ acid composition of Pseudomonas and Alcaligenes $O$ was performed by some workers using gas chroma- 은 tography (Dees and Moss, 1975; Moss et al., 1972; Samuels et al., 1973; Yabuuchi et al., 1974). The Tा results of these analyses show the similarity of the 음 cellular fatty acid composition in the three species of organisms cited above and their possession of $a$ relatively high percentage of 3-hydroxy-tetra- $\vec{\theta}$ decanoicacid.Further investigation on the comparison $\infty$ of fatty acid composition of chlorhexidine-sensitive and -resistant strains of the same species of organisms and on the neutralising effect of specific fatty acid on disinfection by chlorhexidine may explain the relationship between the cellular fatty acid component and resistance to this disinfectant.

$A$. xylosoxidans was resistant to gentamicin and colistin, which were commonly said to be effective against Ps. aeruginosa infection. Prolonged treatment of the patient with these antibiotics may play a significant role in the colonisation and infection? with $A$. xylosoxidans.

We thank Dr E. Yabuuchi for kindly supplying the type strain ATCC-27061 of $A$. xylosoxidans.

\section{References}

Bassett, D. C. J., Stokes, K. J., and Thomas, W. R. G. N (1970). Wound infection with Pseudomonas multi-o vorans: a water-borne contaminant of disinfectant $N$ solution. Lancet, 1, 1188-1191.

Bauer, A. W., Kirby, W. M. M., Sherris, J. C., and ${ }^{\omega}$ Turck, M. (1966). Antibiotic susceptibility testing by a standardized single disk method. American Journal of Clinical Pathology, 45, 493-496.

Calman, R. M., and Murray, J. (1956). Antiseptics in $\stackrel{-}{-}$ midwifery. British Medical Journal, 2, 200-204.

Davies, G. E., Francis, J., Martin, A. R., Rose, F. L., and

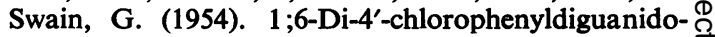
hexane (Hibitane): laboratory investigation of a new $\mathbb{D}$ antibacterial agent of high potency. British Journal $\frac{\circ}{\sigma}$ 
of Pharmacology and Chemotherapy, 9, 192-196.

Dees, S. B., and Moss, C. W. (1975). Cellular fatty acids of Alcaligenes and Pseudomonas species isolated from clinical specimens. Journal of Clinical Microbiology, 1, 414-419.

Ederer, G. M., and Matsen, J. M. (1972). Colonization and infection with Pseudomonas cepacia. Journal of Infectious Disease, 125, 613-618.

Kim, E., Akashi, T., Shimizu, K., and Ohguro, Y. (1973). A contamination of an ultrasonic washing machine with a hibitane resistant organism (in Japanese). Igaku No Ayumi, 85, 355-357.

Møller, V. (1955). Simplified tests for some amino acid decarboxylases and for the arginine dihydrolase system. Acta Pathologica Microbiologica Scandinavica, 36, 158-172.

Moss, C. W., Samuels, S. B., and Weaver, R. E. (1972). Cellular fatty acid composition of selected Pseudomonas species. Applied Microbiology, 24, 596-598.

Samuels, S. B., Moss, C. W., and Weaver, R. E. (1973). The fatty acid of Pseudomonas multivorans (Pseudomonas cepacia) and Pseudomonas kingii. Journal of General Microbiology, 74, 275-279.

Shigeta, S., Higa, K., Ikeda, M., and Endo, S. (1974). A purulent meningitis caused by Achromobacter xylosoxidans (in Japanese). Igaku No Ayumi, 88, 336337.

Yabuuchi, E., and Ohyama, A. (1971). Achromobacter xylosoxidans $\mathrm{n}$ sp. from human ear discharge. Japanese Journal of Microbiology, 15, 477-481.

Yabuuchi, E., Yano, I., Goto, S., Tanimura, E., Ito, T., and Ohyama, A. (1974). Description of Achromobacter xylosoxidans Yabuuchi and Ohyama 1971. International Journal of Systematic Bacteriology, 24, 470477. 